\title{
On the Model Reference Control for Max-Plus Linear Systems
}

\author{
C. A. Maia L. Hardouin R. Santos-Mendes $\quad$ B. Cottenceau
}

\begin{abstract}
This paper deals with the model-reference control of max-plus linear systems. The main contribution of this work is a general control structure that encompasses the previous one found in the literature. It is based on the RST-structure for conventional differentiable system. In addition it provides a comparison among some sub-structures and simulation results are presented.
\end{abstract}

\section{INTRODUCTION}

Several applications in manufacturing, computer and communication systems can be modelled by the so called Discrete Event Systems (DES) theory. These systems are often described by the Petri Net formalism [16]. Timed Event Graphs (TEG) are Timed Petri Nets in which all places have single upstream and single downstream transitions and are used to model DES characterized by delay and synchronization phenomena. TEG can be described by linear equations in the dioid algebra formulation ([2],[8]) and this fact has allowed many important achievements on the modelling and control of DES, as detailed in [6], [15], [7], [13], [10], [11], [9]. TEG control problems are usually stated in a Just-in-Time context, where the design goal is to minimize stocks while guaranteeing performance (e.g. throughput). One possible approach for the control of TEG is the model-reference technique in which a given model describes the desired performance and the design goal is achieved through the calculation of a precompensator or of a feedback controller [7], [13]. The techniques based on feedback control, although favoring stability, are limited in the sense that the reference model must satisfy certain restrictive conditions. Those based on precompensation can guarantee performance for any reference model, but not stability (for the concept of stability in TEG, see [4]). In this context, the present paper proposes a control structure based on well known RST for continuous systems [1] which is a generalization of the work proposed by [7] and [14]. The main advantage of the approach is that it achieves optimality regarding stocks while guaranteeing optimal compliance with any prescribed reference model.

C. A. Maia is with Departamento de Engenharia Elétrica, Universidade Federal de Minas Gerais, Av. Antônio Carlos 6627, Pampulha - 31270-010 Belo Horizonte - MG - Brazil maia@cpdee.ufmg.br

L.Hardouin is with Laboratoire d'Ingénierie des Systèmes Automatisés, Université d'Angers, Av. Notre Dame du Lac 62 - 49000 Angers France laurent.hardouin@istia.univ-angers.fr

R. Santos-Mendes is with Faculdade de Engenharia Elétrica e de Computação, Universidade Estadual de Campinas, Brazil. rafael@dca.fee.unicamp.br

B. Cottenceau is with Laboratoire d'Ingénierie des Systèmes Automatisés, Université d'Angers, France bertrand.cottenceau@istia.univ-angers.fr
The paper is organized as follows. Section II introduces some algebraic tools concerning the Dioid and Residuation theories and their applications to max-plus linear system. Section III introduces some control results and develops the proposed control structure. Simulation Results are shown in section IV. A conclusion is given in section $\mathrm{V}$.

\section{MAX-PLUS LINEAR SYSTEM THEORY}

An important framework to deal with max-plus systems is the dioid algebra. A dioid $\mathcal{D}$ is an idempotent semiring. The two internal operations are denoted $\oplus$ and $\otimes$. The operation $\oplus$ is associative, commutative and idempotent, that is, $a \oplus$ $a=a, \forall a \in \mathcal{D}$. The operation $\otimes$ is associative (but not necessarily commutative) and distributive at left and at right with respect to $\oplus$. The neutral elements of $\oplus$ and $\otimes$ are represented by $\varepsilon$ and $e$ respectively, and $\varepsilon$ is absorbing for $\otimes(\forall a \in \mathcal{D}, \varepsilon \otimes a=a \otimes \varepsilon=\varepsilon)$. In a dioid, a partial order relation is defined by $a \succeq b$ iff $a=a \oplus b$ and $x \wedge y$ denotes the greatest lower bound between $x$ and $y$. A dioid $\mathcal{D}$ is said to be complete if it is closed for infinite $\oplus$-sums and if $\otimes$ distributes over infinite $\oplus$-sums. Most of the time the symbol $\otimes$ will be omitted as in conventional algebra, moreover, $a^{i}=a \otimes a^{i-1}$ and $a^{0}=e$.

The least solution of the equation $x=a x \oplus b$ is a relevant issue in many max-plus linear systems problems and an important result is presented in theorem 1.

Theorem 1 ( [2], th. 4.75): The implicit equation $x=$ $a x \oplus b$ defined over a complete dioid $\mathcal{D}$, admits $x=a^{*} b$ as least solution, where $a^{*}=\bigoplus_{i \in \mathbb{N}} a^{i}$ (Kleene star operator).

Automatic control synthesis involves the inversion of mappings. Mappings defined over dioid does not always admit inverse, but the residuation theory allows to characterize solution set of inequalities such that $f(x) \preceq y$. The reader may consult [3] to obtain an exhaustive presentation of this theory.

Definition 1 (Residual and residuated mapping): An isotone mapping $f: \mathcal{D} \rightarrow \mathcal{E}$, where $\mathcal{D}$ and $\mathcal{E}$ are partially ordered sets, is a residuated mapping if for all $y \in \mathcal{E}$ there exists a greatest solution for the inequality $f(x) \preceq y$ (hereafter denoted $f^{\sharp}(y)$ ). The mapping $f^{\sharp}$ is called the residual of $f$.

The mappings $L_{a}: x \mapsto a \otimes x$ and $R_{a}: x \mapsto x \otimes a$ defined over a complete dioid $\mathcal{D}$ are both residuated ([2], p. 181). Their residuals are isotone mappings ${ }^{1}$ denoted respectively by $L_{a}^{\sharp}(x)=a \phi x$ and $R_{a}^{\sharp}(x)=x \phi a$. Some useful dioid

${ }^{1} f$ is an isotone mapping if it preserves order, that is, $a \preceq b \Longrightarrow f(a) \preceq$ $f(b)$. It is an antitone mapping if it reverses the order, that is, $a \preceq b \Longrightarrow$ $f(b) \preceq f(a)$. 
formulæ involving these residuals are given below.

$$
\begin{aligned}
a(a \phi x) & \preceq x \\
a \phi(a x) & \succeq x \\
a(a \phi(a x)) & =a x \\
a ф a & =(a ф a)^{*} \\
a(b a)^{*} & =(a b)^{*} a \\
s^{*} \preceq a^{*} & \Leftrightarrow s \preceq a^{*} \\
(a b) \phi x & =b \phi(a \phi x) \\
a \phi(a(a \phi x)) & =a \phi x
\end{aligned}
$$

A trajectory of a TEG transition $x$ is a firing date sequence $\{x(k)\} \in \mathbb{Z}$. For each increasing sequence $\{x(k)\}$, it is possible to define the transformation $x(\gamma)=\bigoplus_{k \in \mathbb{Z}} x(k) \gamma^{k}$ where $\gamma$ is a backward shift operator in event domain (that is $y(\gamma)=\gamma x(\gamma) \Leftrightarrow\{y(k)\}=\{x(k-1)\}$, see [2], p. 228). This transformation is analogous to the $Z$-transform used in discrete-time classical control theory and the formal series $x(\gamma)$ is a synthetic representation of the trajectory $x(k)$. The set of the formal series in $\gamma$ is denoted by $\overline{\mathbb{Z}}_{\max } \llbracket \gamma \rrbracket$ and constitutes a dioid. In general, MIMO max-plus linear System can be represented by

$$
\begin{aligned}
x & =A x \oplus u \\
y & =C x
\end{aligned}
$$

where vectors $x \in\left(\overline{\mathbb{Z}}_{\max } \llbracket \gamma \rrbracket\right)^{n}, u \in\left(\overline{\mathbb{Z}}_{\max } \llbracket \gamma \rrbracket\right)^{p}$ and $y \in$ $\left(\overline{\mathbb{Z}}_{\max } \llbracket \gamma \rrbracket\right)^{m}$ represent respectively, the state, the input and output trajectories of the system and $A, B, C$ are the system matrices of appropriate dimmensions. These trajectories can be related ([2], p. 243) by the equation $y=H u$, where $H=C A^{*} B \in\left(\overline{\mathbb{Z}}_{\max } \llbracket \gamma \rrbracket\right)^{m \times p}$ is called the transfer matrix of the TEG. Entries of matrix $H$ are periodic series ([2], p. 260) in the dioid $\overline{\mathbb{Z}}_{\max } \llbracket \gamma \rrbracket$. These series are usually represented by $p(\gamma) \oplus q(\gamma)\left(\tau \gamma^{\nu}\right)^{*}$, where $p(\gamma)=\bigoplus_{i=0}^{n-1} \quad p_{i} \gamma^{i}, p_{i} \in$ $\mathbb{N}$, is a polynomial that represents the transient and $q(\gamma)=$ $\bigoplus_{j=0}^{\nu-1} q_{j} \gamma^{j}, q_{i} \in \mathbb{N}$ is a polynomial that represents a pattern which is repeated each $\tau$ time units and each $\nu$ firings of the transition.

The asymptotic slope of a periodic series $s=p(\gamma) \oplus$ $q(\gamma)\left(\tau \gamma^{\nu}\right)^{*}$, denoted $\sigma_{\infty}(s)$, is defined as the ratio $\sigma_{\infty}(s)=$ $\frac{\nu}{\tau}$, calculated in the conventional algebra. In a manufacturing context, the asymptotic slope can be viewed as the production rate of the system.

\section{CONTROL METHOD}

The control method proposed herein is based on the Justin-Time strategy and on the model reference approach [7] and is summarized as follows. First let $H \in\left(\overline{\mathbb{Z}}_{\max } \llbracket \gamma \rrbracket\right)^{m \times p}$ be the transfer matrix of the plant and $G_{r e f} \in\left(\overline{\mathbb{Z}}_{\max } \llbracket \gamma \rrbracket\right)^{m \times p}$ be the reference model, i.e., the desired transfer matrix for the controlled system. In the model reference context, the aim is to delay as much as possible the firing dates of the input transition by assuring that the controlled system transfer function be less than or equal to the reference model (which represents in a manufacturing context the restriction imposed by the customers demand). This idea is illustrated in the figure 1. In this figure, $u, y$ and $H$ represents respectively the input, the output and the system transfer function; the reference model is given by $G_{r e f}$ and the external input ${ }^{2}$ is given by $v$. The control problem is presented in the following. Let $H \in\left(\overline{\mathbb{Z}}_{\max } \llbracket \gamma \rrbracket\right)^{m \times p}$ be the matrix transfer function of the system, $G_{y v} \in\left(\overline{\mathbb{Z}}_{\max } \llbracket \gamma \rrbracket\right)^{m \times p}$ be the controlled system transfer function and $G_{r e f} \in\left(\overline{\mathbb{Z}}_{\max } \llbracket \gamma \rrbracket\right)^{m \times p}$ be a reference model i.e., the desired transfer function for the controlled system. So the problem can be stated as follows.

Given a TEG, which controller will assure the maximal transfer function between $u$ and $v\left(G_{u v}\right)$ such that $G_{y v} \preceq$ $G_{r e f}$ ? Considering the just-in-time context, the goal is to obtain a controller satisfying the reference specification $\left(G_{y v} \preceq G_{r e f}\right)$ while delaying as much as possible the product admissions into the system. In this sense, the transfer function $G_{u v}$ is the one which leads to the maximal control trajectory for a given input $v$.

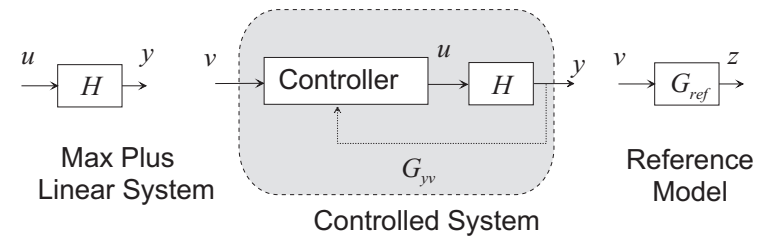

Fig. 1. Model Reference Control

\section{A. RST-structure control}

It is known that the existence of the greatest feedback controller depends on the model reference choice [7]. A control structure based on a precompensator and a feedback controller improves the controlled system performance, as shown in [14]. This approach always lead to an optimal behavior of the closed-loop system and assures better robustness properties than the simple feedback [12].

In this section, a more general structure is proposed based on the well known RST-structure for continuous systems. This control approach is based on three control matrices as illustrated in the figure 2. By using figure 2, one has the

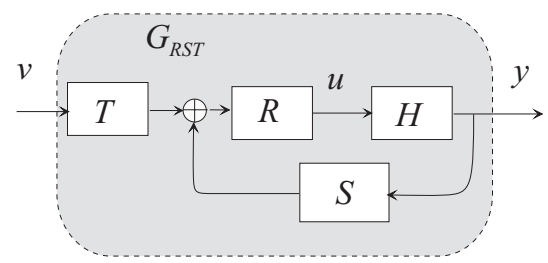

Fig. 2. RST-structure Control

following control law

$$
u=R(T v \oplus S y) .
$$

\footnotetext{
${ }^{2}$ In a Just-in-Time context, $v$ represents the available supply of raw material and $u$ represents the allowance of the raw material into the system.
} 
Therefore $y=H u=H R T v \oplus H R S y$, then theorem 1 and formulae (5) yield to the following equations.

$$
\begin{aligned}
y & =(H R S)^{*} H R T v=H(R S H)^{*} R T v \\
& =G_{R S T} v \\
u & =(R S H)^{*} R T v .
\end{aligned}
$$

So the objective is to obtain matrices $R, S, T$ that maximize $u$ with the restriction that $G_{R S T} \preceq G_{r e f}$, where $G_{r e f}$ is the model reference. As in [14], one can show that:

$$
\begin{array}{ll} 
& H(R S H)^{*} R T \preceq G_{r e f} \\
\Leftrightarrow \quad & (R S H)^{*} R T \preceq H \phi G_{r e f} \\
\Leftrightarrow \quad & H(R S H)^{*} R T \preceq H(H ф G r e f) .
\end{array}
$$

From these inequalities, one can solve the control problem by observing that matrices $R$ and $T$ must satisfy:

$$
R T \preceq H \phi G_{r e f} .
$$

Actually the upper bound $H \phi G_{r e f}$ is always reachable; for instance take $R=I^{p \times p}, S=[\varepsilon]_{p \times m}$ and $T=H \phi G_{r e f}$, which correspond to the precompensator case. In this case, by taking equation (14) and expression (15) into account, the control action is given by $\left(H \nmid G_{r e f}\right) v$, and it is maximized since $u \preceq\left(H \nmid G_{r e f}\right) v$. Therefore it is important to observe that one possible solution for the proposed control problem is achieved if one takes, $R T=H ф G_{r e f}$. By considering this particular solution, the expression (15) assures the following developments.

$$
\begin{aligned}
& H(R S H)^{*}\left(H \nmid G_{r e f}\right) \preceq H\left(H \nmid G_{r e f}\right) \\
& \Leftrightarrow \quad(H R S)^{*} H\left(H \phi G_{r e f}\right) \preceq H\left(H \nmid G_{r e f}\right) \\
& \Leftrightarrow \quad(H R S)^{*} \preceq\left(H\left(H \nmid G_{r e f}\right)\right) \phi\left(H\left(H \nmid G_{r e f}\right)\right) \\
& \Leftrightarrow \quad H R S \preceq\left(H\left(H \nmid G_{r e f}\right)\right) \phi\left(H\left(H \nmid G_{r e f}\right)\right)
\end{aligned}
$$

The expression (17) is a consequence of equation (5); (18) is a result of the residuation definition and (19) comes from (4) and (6).

As a result, the RST control problem can be solved if $R T=H ф G_{r e f}$ with the restriction given by the inequality (19). Synthetically, an optimal control is obtained if

$$
\begin{aligned}
R T & =H \phi G_{r e f} \\
H R S & \preceq\left(H\left(H \phi G_{r e f}\right)\right) \phi\left(H\left(H \nmid G_{r e f}\right)\right) .
\end{aligned}
$$

In general, the calculation of matrices $R, S$ and $T$ is, in some extent, equivalent to solve the diophantine equation for conventional linear continuous systems. This task can be easily done by using some particular control structures of the proposed RST-structure. These cases are presented and discussed below.

\section{B. R-Structure}

This case is known in literature as precompensation approach [5]. In this structure, the controlled system is simplified by considering that $T=I^{p \times p}$ and $S=[\varepsilon]_{p \times m}$. So the problem is solved by finding the greatest precompensator
$R$ such that $H R \preceq G_{r e f}$. The optimal solution, denoted by $R_{\text {opt }}$, is obtained directly from equation (20):

$$
R_{o p t}=H \phi G_{r e f} .
$$

This result means that, for a given external input $v \in$ $\left(\overline{\mathbb{Z}}_{\max } \llbracket \gamma \rrbracket\right)^{p}$, the input vector, given by $u=R v$, will be maximal. In fact, for any $R$ such that $H R \preceq G_{r e f}, R \preceq$ $R_{o p t}$, therefore the isotony property assures that $u=R v \preceq$ $R_{o p t} v$. However, since it is an open-loop approach, it does not reinforce the stability ${ }^{3}$ of the system and is not efficient when disturbances act on the system( see [14] for details).

\section{S-Structure}

In this case one sets matrices $R$ and $T$ equal to identity and it is known as feedback approach. By using equation (13), the closed-loop transfer matrix between $y$ and $v$, for a given feedback controller $S$, is given by $H(S H)^{*}$. Therefore the problem is solved by finding the greatest $S$ such that $H(S H)^{*} \preceq G_{r e f}$. This problem can be solved via residuation theory if some restrictions are imposed on the reference model. The following result is due to [7].

Proposition 1: Let $H \in\left(\overline{\mathbb{Z}}_{\max } \llbracket \gamma \rrbracket\right)^{m \times p}$ be the transfer function of a TEG. For every reference model $G_{r e f}$ such that $G_{\text {ref }}=E^{*} H$ or $G_{r e f}=H F^{*}\left(\right.$ where $E \in\left(\overline{\mathbb{Z}}_{\max } \llbracket \gamma \rrbracket\right)^{m \times m}$ and $\left.F \in\left(\overline{\mathbb{Z}}_{\max } \llbracket \gamma \rrbracket\right)^{p \times p}\right)$ there exists a greatest feedback controller $S \in\left(\overline{\mathbb{Z}}_{\max } \llbracket \gamma \rrbracket\right)^{p \times m}$ such that the transfer function of the closed-loop system is less than or equal to $G_{r e f}$. The greatest feedback controller is:

$$
S_{\text {opt }}=H \phi G_{r e f} \phi H .
$$

One should remark from equation (14) that in this strategy the transfer function between $u$ and $v$ is $(S H)^{*}$.

Property 1: If $H(S H)^{*} \preceq G_{r e f}$, for given $H$ and $G_{r e f} \in$ $\left(\overline{\mathbb{Z}}_{\max } \llbracket \gamma \rrbracket\right)^{m \times p}$, then $(S H)^{*} \preceq R_{\text {opt }}=H \phi G_{\text {ref }}$.

The proof comes from the fact that $a x \preceq b \Leftrightarrow x \preceq a \oint b$. This property means that the transfer function between $u$ and $v$ for the optimal open-loop strategy is always greater than or equal to the one obtained for any feasible feedback strategy.

It is important to remark that under some restriction on the reference model the simple feedback (S-structure) is better that (R-structure) in the stabilization context [14], [7]. However the precompensation always leads to an optimal control action which is equal to $R_{o p t} v$ and there is no restriction concerning the reference model choice (see subsection IIIB). The next structure to be presented exploits the main advantages of these two approaches.

\section{RS-structure}

In [14], it was considered a particular case where the precompensator $T$ is equal to the identity matrix. This structure is shown in figure 3. In this case the direct application of residuation theory to the system given by (20) and (21) leads to optimal controller matrices given by:

$$
\begin{aligned}
R_{o p t} & =H \nmid G_{r e f}, \\
S_{o p t} & =\left(H R_{o p t}\right) \phi\left(H R_{o p t}\right) \phi\left(H R_{o p t}\right) .
\end{aligned}
$$

\footnotetext{
${ }^{3}$ Stability here means that the number of tokens of system is bounded.
} 


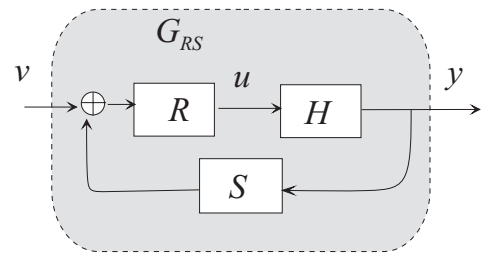

Fig. 3. RS-structure Control

\section{E. ST-structure}

This is a new control structure. It is obtained from the RST one by simply making matrix $R$ equal to the identity. It is illustrated in the figure 4.

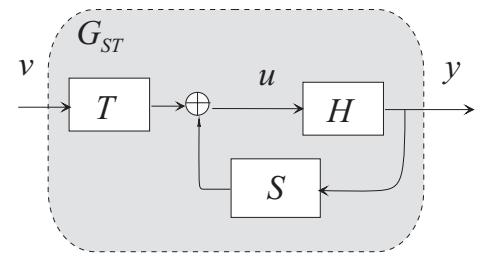

Fig. 4. ST-structure Control

This approach always leads to an optimal control structure. Actually, since $R=I^{p \times p}$, by using system given by (20) and (20) , one can easily show that the optimal matrices are

$$
\begin{aligned}
T_{o p t} & =H \phi G_{r e f} \\
S_{\text {opt }} & =H \phi\left(H T_{o p t}\right) \phi\left(H T_{o p t}\right)
\end{aligned}
$$

At this point, it is known that both RS-structure and STstructure lead to an optimal control structure. Therefore, one question arises: what are the differences between them? One difference is pointed out in the property below.

Property 2: For the ST structure, the feedback from the output $y$ to the input $u$ is greater than or equal the one obtained for the RS-structure.

Proof: By inspection of figures 4 and 3 one can see that it suffices to show that the feedback given by equation (26) is greater than or equal the product of equation (23) by equation (24). In fact, by using equation (7), equation (24) can be rewritten as: $\left(H R_{o p t}\right) \phi\left(H R_{o p t}\right) \phi\left(H R_{o p t}\right)=$ $R_{o p t} \phi\left(H \phi\left(H R_{o p t}\right)\right) \phi\left(H R_{o p t}\right)$. By equation (23), $R_{\text {opt }}=H \phi G_{\text {ref }}$, so equation (8) leads to: $\left(H R_{o p t}\right) \phi\left(H R_{o p t}\right) \phi\left(H R_{o p t}\right)=R_{o p t} \phi R_{o p t} \phi\left(H R_{o p t}\right)$. Therefore, for the RS-structure, one uses equation (1) to obtain $R_{o p t} S_{o p t} \preceq R_{o p t} \phi\left(H R_{o p t}\right)$.

In contrast, for the ST-structure, the feedback is given by $H \phi\left(H T_{o p t}\right) \phi\left(H T_{o p t}\right)$, where $T_{o p t}=H \phi G_{r e f}$. So expression (2) assures that $H \phi\left(H T_{o p t}\right) \succeq T_{o p t}$. By isotony, $H \phi\left(H T_{o p t}\right) \phi\left(H T_{o p t}\right) \succeq T_{o p t} \phi\left(H T_{o p t}\right)$. One concludes the proof by remembering that $T_{o p t}=R_{o p t}$.

Remark 1: This results may suggest that the ST-structure is more favorable to stability than the $\mathrm{RS}$ one since its greatest feedback leads, in the worst case, to the same number of arcs (from output to input) of the RS one. Concept of stability here means that the number of tokens of the closed-loop system remains bounded and one must remember that a strongly connected graph is always stable. For more detail regarding stability see [2] and [4].

\section{Simulation Results}

The objective here is to illustrate the application of model reference approach to control a max-plus linear system represented by a Timed Event Graph.

Consider the TEG depicted in gray box of the Fig. 5 as an example. It models a workshop with 3 machines ( $M_{1}$ to $M_{3}$ ). Machines $M_{1}$ and $M_{2}$ can process parts which are assembled by machine $M_{3}$. Inputs $u_{1}$ and $u_{2}$ represent the admission dates of parts into the system; the transportation time is 1 for both parts; machine $M_{1}$ can process one part in 2 time units; machine $M_{2}$ can process one part in 3 time units. The transportation time between machines $M_{1}$ and $M_{3}$ is 4 time units and between machines $M_{2}$ and $M_{3}$ is 5 time units. Machine $M_{3}$ can assemble one part in 4 time units, the transportation time until the output is 6 time units and $y$ represents the output date of the final product. As discussed in section II, one must remind that this system can be modelled by equations (9) and (10) and the input-output relationship is given by the equation $y=H u$, where $H$ is the transfer function. Therefore appropriated computations ${ }^{4}$ lead to:

$$
y=\left[17(4 \gamma)^{*} \quad 19(4 \gamma)^{*}\right] u
$$

The obtained relationship means that the transfer functions between $y$ and $u_{1}$ and $u_{2}$ have a maximum production rate of $1 / 4$. This is a direct consequence of the workshop configuration since the machine $M_{3}$ has the slowest production rate (actually, it is the bottleneck for the system).

To compare RS and ST control strategies, the reference model is chosen as $G_{r e f}=\left[17(4 \gamma)^{*} 19(5 \gamma)^{*}\right]$. That is, the objective is to keep the production rate between $y$ and $u_{1}$ unchanged but to allow the one between $y$ and $u_{2}$ to reduce until $1 / 5$. The obtained results are given below.

\section{A. RS-structure}

By using equations (23) and (24), one obtains the solution: $R_{o p t}=\left(\begin{array}{cc}(4 \gamma)^{*} & 2(5 \gamma)^{*} \\ 2 \gamma(4 \gamma)^{*} & (5 \gamma)^{*}\end{array}\right)$ and $S_{o p t}=\left(\begin{array}{lll}3 \gamma^{5}(4 \gamma)^{*} & \varepsilon\end{array}\right)^{t}$. Figure 5 shows one realization of the controlled workshop system.

\footnotetext{
${ }^{4}$ Softwares to handle dioid algebra using Scilab can be downloaded from the site [17].
} 


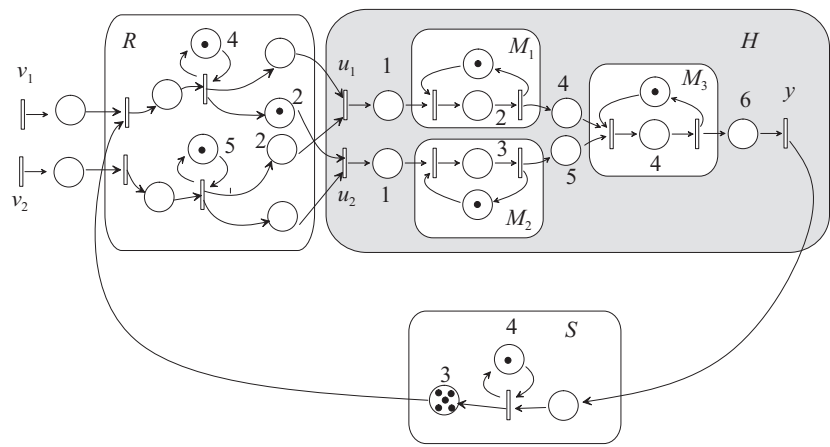

Fig. 5. Controlled Workshop System with the RS-strategy.

\section{B. ST-structure}

By using the equations (25) and (26), one obtains the following solution: $T_{o p t}=\left(\begin{array}{cc}(4 \gamma)^{*} & 2(5 \gamma)^{*} \\ 2 \gamma(4 \gamma)^{*} & (5 \gamma)^{*}\end{array}\right)$ and $S_{\text {opt }}=$ $\left(3 \gamma^{5}(4 \gamma)^{*} \quad 1 \gamma^{5}(4 \gamma)^{*}\right)^{t}$. Figure 6 shows one realization of the controlled workshop system.

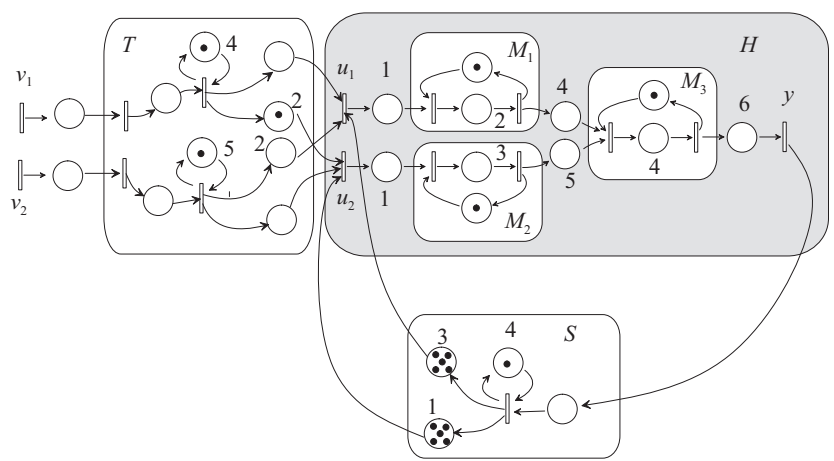

Fig. 6. Controlled Workshop System with the ST-strategy.

Remark 2: Both RS and ST approaches lead to an optimal closed-loop system in a just-in-time sense, that is, the control action is maximized. However in this example calculation results in $R_{o p t} S_{o p t}=\left(3 \gamma^{5}(4 \gamma)^{*} 5 \gamma^{6}(4 \gamma)^{*}\right)^{t}$ for the RSstrategy . The obtained feedback matrix for the ST-strategy is $\left(3 \gamma^{5}(4 \gamma)^{*} 1 \gamma^{5}(4 \gamma)^{*}\right)^{t}$. By remembering that $1 \gamma^{5}(4 \gamma)^{*}=$ $1 \gamma^{5} \oplus 5 \gamma^{6}(4 \gamma)^{*}$, the feedback from the output $y$ to the input $u$ is greater than the one obtained for the RS-structure. This is a consequence of property 2 .

\section{CONCLUSIONS AND FUTURE WORKS}

The main contribution of this paper is a general structure for the model-reference control of DES described by the max-plus algebra. The approach is based on the well known RST-structure control for conventional differentiable system. The presented results encompasses the ones found in the literature and some comparisons concerning performance and stability among particular cases are made. An important issue is to investigate robustness of the proposed structure, as initiated by [12]. Another step is the control design in the uncertain environment as proposed by [11] for the simple feedback case.

\section{REFERENCES}

[1] K.J Astrom, and B. Wittenmark, Computer Controlled Systems, Prentice Hall, Englewood Cliffs, 1990.

[2] F. Baccelli, G. Cohen, G. Olsder, and J. Quadrat, Synchronisation and Linearity: An Algebra for Discrete Event Systems, John Wiley and Sons, New York, 1992.

[3] T. Blyth and M. Janowitz, Residuation Theory, Pergamon Press, Oxford, 1972.

[4] C. Commault, Feedback stabilization of some event graph models, IEEE Trans. Automat. Contr., vol. 43, no. 10, pp. 1419-1423, October 1998.

[5] B. Cottenceau. Contribution à la commande de systèmes à événements discrets : synthèse de correcteurs pour les graphes d'événements temporisés dans les diö̈des, Thèse de doctorat, Université d'Angers, Angers - France, 1999.

[6] B. Cottenceau, L. Hardouin, J. Boimond, and J. Ferrier, Synthesis of greatest linear feedback for timed event graphs in dioid, IEEE Trans. Automat. Contr., vol.44, no. 6, pp. 1258-1262, June 1999.

[7] B. Cottenceau, L. Hardouin, J. Boimond, and J. Ferrier, Model Reference Control for Timed Event Graphs in Dioid, Automatica, vol. 37, pp. 1451-1458, 2001.

[8] S. Gaubert. Théorie des systèmes linéaires dans les dioïdes, Thèse de doctorat, Ecole des Mines de Paris, Paris, 1992.

[9] L. Hardouin. Sur la Commande linéaire de Systèmes à Evenements Discrets dans l'algèbre (max,+), Habilitation à diriger des recherches, Université d'Angers, Angers - France, 2004.

[10] S. Lahaye, J. Boimond, and L. Hardouin, Linear periodic systems over dioids, Discrete Event Dynamic Systems. Theory and Applications, vol. 14, pp. 133-152, 2004.

[11] M. Lhommeau, L. Hardouin, B. Cottenceau, and L. Jaulin, Interval analysis in dioid : application to obust controller design for timed event graphs. Automatica, vo. 40, no.11, pp. 1923-1930, Nov. 2004,

[12] M. Lhommeau, L. Hardouin, C. A. Maia, and R. Santos-Mendes, Control and robustness analysis for $(\max ,+)$ linear systems. In Workshop On Discrete Event Systems (WODES'2004), Reims, France, Sept. 2004.

[13] R. Lüders and R. Santos-Mendes, Generalized Multivariable Control of Discrete Event Systems in Dioid, In 6th International Workshop on Discrete Event Systems (WODES'02), Zaragoza, Spain, 2002.

[14] C. A. Maia, L. Hardouin, R. Santos-Mendes, and B. Cottenceau, Optimal Closed-loop of Timed Event Graphs in Dioids, IEEE Trans. Automat. Contr., vol. 48, no. 12, pp. 2284-2287, Dec. 2003.

[15] E. Menguy, J. Boimond, L. Hardouin, and J. Ferrier, Just-in-time Control of Timed Event Graphs: Update of Reference Input, Presence of Uncontrollable Input, IEEE Trans. Automat. Contr., vol. 45, no. 11, pp. 2155-2158, 2000.

[16] T. Murata, Petri nets : properties, analysis and applications, Proceedings of the IEEE, vol. 77, no.4, pp. 541-580, 1989.

[17] SW2005. Software Tools for Manipulating Periodic Series, Jan. 2005. http://www.istia-angers.fr/hardouin/outils.html 